\title{
Demographic findings of 104 patients with percutaneous endoscopic gastrostomy
}

\author{
Mustafa Serdar Cantez, Nelgin Gerenli, Vildan Ertekin, Özlem Durmaz \\ Istanbul University, Istanbul Medical Faculty, Division of Pediatric Gastroenterology Hepatology and Nutrition, Istanbul, Turkey
}

\begin{abstract}
Summary
Aim: The aim of this study was to evaluate demographic findings of 104 children who had undergone percutaneous endoscopic gastrostomy insertion procedure.

Material and Method: Children who had undergone PEG insertion procedure between 2005 and 2012 were included in the study. Demographic data were collected retrospectively from the clinic charts.

Results: A hundred and four patients (44 girls, 46\%) with 269 percutaneous endoscopic gastrostomy and percutaneous gastrostomy related procedures in the last 7 years were enrolled in this study. The mean follow-up time was 36.5 months (3-84 months). Patients with neurological disabilities and metabolic diseases constituted the majority (45\% and $40 \%$ respectively). The median age of patients with percutaneous endoscopic gastrostomy procedure was 5.9 years ( 2 months - 16 years) and the mean weight was $17.7 \mathrm{~kg}$ ( $3-47 \mathrm{~kg}$ ). The median weight $Z$ score before PEG procedure was -1.98. The follow-up weight $Z$ scores were available for 45 patients. The follow-up $z$ score was 1,21 after a mean period of 18 months following the PEG procedure $(p=0.0007)$. Major complications were observed in 3 patients (\%2.8). Minor complications were observed in 10\% of all procedures. Skin complications were observed in 13 patients (12.5\%). Local infections in the stoma site were the most common skin complications (7/13). Systemic infections were not observed.

Conclusions: Children with metabolic diseases constituted a major subgroup in our cohort. This finding is an important difference of our cohort compared to other European pediatric series in which primary neurological disabilities were the main indication for PEG. Despite complications, PEG procedure seems to be safe for supporting children with nutritional problems. (Turk Arch Ped 2013; 48: 210-4)
\end{abstract}

Key words: Child, demographic findings, percutaneous endoscopic gastrostomy

\section{Introduction}

Percutaneous endoscopic gastrostomy (PEG) is an invasive procedure which has started to be used more frequently in recent years in patients who experience feeding problems. The procedure of percutaneous endoscopic gastrostomy was performed by Gauderer (1) in 1980 for the first time in the world. Since that time PEG has been also used in the area of pediatric gastroenterology. PEG which can be easily placed in experienced hands provides efficient feeding in care and treatment of neurologic, metabolic, oncologic diseases as well as other gastrointestinal diseases which necessitate direct access to the stomach by surpassing the mouth, pharynx and esophagus including anatomic and functional disorders of the upper gastrointestinal tract. In our center, the procedure of PEG is being performed since 2005. In this study, we aimed to examine the demographic data and complication rates in children who had undergone PEG.

\section{Material and Method}

In this study, the data of our patients who underwent PEG procedure in our unit between 2005 and 2012 were examined retrospectively. The demographic properties of the patients, the units in which they were being followed up and complications developed in relation with the procedure were obtained from the information bank of the endoscopy unit and patient files. A standard PEG set (Nutricia Flocare ${ }^{\circledR}$ PEG set No: $10,12,14)$ was placed initially in all patients. 
The standard PEG set is a set which absolutely requires endoscopic procedure and which can only be exchanged by endoscopic intervention. The components of endoscopy set can be briefly summarized as feeding connection, transparent tube with varying lengths, openable clamp, a safety cap which fixes the tube, external silicone pulling disc and internal silicone pulling disc which provide fixing and twisting of the tube without breaking (Figure 1). Button PEG is a PEG set which the family itself can exchange at home, which can be fixed inside the stomach due to its inflatable silicone balloon, which is maintained on the surface of the skin and which has no tube (Figure 2). The procedure of standard PEG placement which is also used currently was developed by Keymling (2) as the 'pull - through' technique after being defined by Gauderer (1) for the first time. Standard percutaneous endoscopic gastrostomy sets were exchanged yearly unless any problem occured and button PEG tubes were exchanged every 3 months. In all patients, sedo-analgesia was provided using midazolam $(0.1 \mathrm{mg} /$ $\mathrm{kg}$, maximum $5 \mathrm{mg}$ ) and meperidine $(1 \mathrm{mg} / \mathrm{kg}$, maximum $50 \mathrm{mg}$ ) and intravenous prophylactic cephazoline was administered before and after the prodecure for 24 hours (25-50 mg/kg/day).

\section{Results}

PEG was performed in 104 patients between January 2005 and September 2012 (46 girls, 44\%). The median age was found to be 5.9 (2 months-16 years) in the patient group who underwent PEG. The mean weight was found to be $17.7 \mathrm{~kg}$ (ranges $3-47 \mathrm{~kg}$ ). The median weight $Z$ score before PEG was found to be -1.98 (ranges: $-12-3.55$ ) in the patients. Follow-up weight values could be reached in 45 of 104 patients included in our patient group. The median Z score after a mean period of 18 months following PEG was found to be 1,21 in these 45 patients. A significant difference was found, when the $Z$ scores for weight before and after the procedure were compared using t-test $(p=0.0007)$. 269 interventions were performed in 104 patients included in our patient group. These interventions include primary PEG procedure, exchange of PEG tube, switching to button PEG and switching from button PEG to standard PEG. In 16 of our patients (15\%), switching from standard PEG to button PEG was performed. The reason for switching from standard PEG to button PEG was the fact that button PEG was found to be more convenient for all patients. A total of 89 procedures were performed in patients who were using button PEG. Switching from button PEG to standard PEG was performed in two patients $(2 \%)$. The distribution of the procedures is summarized in Graphic 1. The patients were referred to the following units: Division of Pediatric Neurology: $n=47(45 \%)$, Division of Metabolism and Nutrition: $\mathrm{n}=42(40 \%)$, Pediatric Intensive Care Unit: $\mathrm{n}=6$ $(6 \%)$ and others: $n=9(8.6 \%)$. The distribution of the others is as follows: External center: three patients, Neonatal Intensive Care Unit: two patients, Emergency Department: two patients, Bone Marrow Transplantation Unit: one patient and Otolaryngology Clinic: one patient. The distribution of the patients with neurologic and metabolic diseases is shown in Graphic 2 and 3.

Significant complications related with percutaneous endoscopic gastrostomy were defined as mortality, prolongation of hospitalization compared to the normal hospitalization time and conditions which required additional intervention. Significant complications were observed in three patients $(2.8 \%)$. These included gastrocolic fistula, esophageal tear and cardiorespiratory arrest. Gastrocolic fistula was manifested four months after the procedure and was corrected surgically. Esophageal tear developed during traction of a $10 \mathrm{~F}$ PEG catheter in relation with the catheter disc in a 2-month-old patient with severe neurologic problems transferred from the neonatal intensive care unit and the patient was lost on the second day, although surgical correction was performed. The case of cardiorespiratory arrest which was the last significant complication of this series developed just after the procedure in a 5-year-old female patient who had a large rhabdomyosarcoma in the neck and who was transferred from the Otolaryngology clinic following sedoanalgesia. The patient was intubated and followed up in

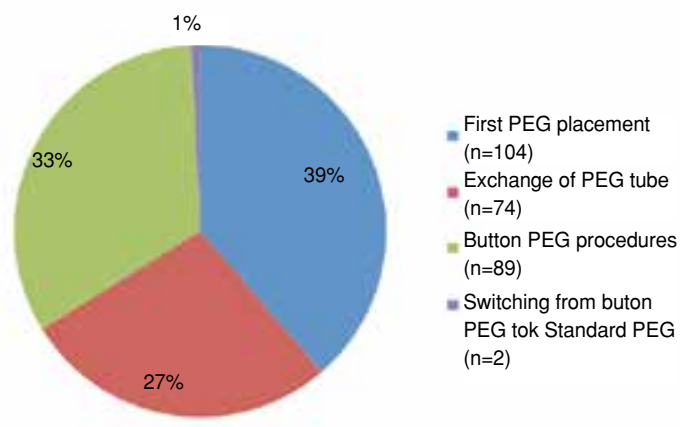

Graphic 1. Distribution of PEG procedures

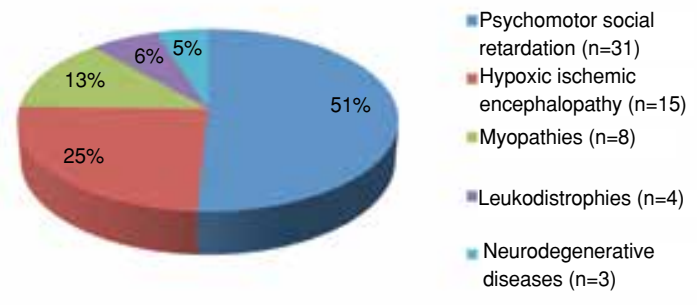

Graphic 2. Distribution of neorology patients 
the Intensive Care Unit. Afterwards, she was transferred to the Hematology ward and her chemotherapy was completed. Following chemotherapy the PEG catheter was removed, since the necessity was eliminated and she was discharged.

Mild complications were observed with a rate of $10 \%$ during all procedures. While the rate of complication was $3.3 \%$ in standard gastrostomies, it was found to be $26.3 \%$ in button gastrostomies. The complications which developed in patients who had undergone button PEG were observed as mild complications related with deformation of button PEG and deflation of the balloon or inability to deflate the balloon in all patients. Cutaneous complications related with PEG were observed in 13 patients and its frequency was found to be $12.5 \%$. Infection was observed in 7 of these 13 patients; the results of ten wound swab cultures obtained from 7 patients were as follows: Pseudomonas was grown in 6 culture samples and dual microorganisms were grown in the remaining four culture samples. The patients were cured with appropriate antibiotic treatment without any problem. Removal of percutaneous endoscopic gastrostomy was performed in three patients. In one of the patients in whom removal of percutaneous endoscopic gastrostomy was performed, the necessity of continuous feeding was eliminated. In another patient, percutaneous endoscopic gastrostomy was removed with the request of the patient and family. In the third patient (the abovementioned patient with rhabdomyosarcoma), PEG was removed following completion of chemotherapy. The general demographic data of the patients are summarized in Table 1.

\section{Discussion}

The procedure of percutaneous endoscopic gastrostomy has been used with success since it was defined by Gauderer et al (1) in 1980 for the first time. In the article of Gauderer (3) which was published about 20 years later after the first definition of PEG in 1980 and which explained the historical process of PEG, the author reported that his first article had been referred for 483 times, 216000 PEGs were placed in USA yearly and 12 commercial companies started to produce PEG. Percutaneous endoscopic gastrostomy is a significant intervention which provides direct access to the stomach for foods surpassing the part from the mouth to the stomach in patients who experience feeding problems in conditions where oral feeding and/ or feeding by nasogastric tube is risky. 104 patients has benefited from PEG in our clinic since 2005. When the distribution of 104 patients was examined, it was observed that the patients with neurologic problems were in the first order. This is compatible with large series published in the world $(4,5)$. In contrast, patients with metabolic diseases were in the second order in our patient group and these patients constituted almost a large group as observed with the patients with neurologic diseases. This finding is noted and shows difference, when compared with some of the large series published previously (5). In the study performed by Srinivasan et al. (5), 384 PEGS were placed and a total of 601 PEG-related procedures were performed. Among the 384 patients presented in this study, the number of patients with neurologic problems was 160 , whereas the number of patients with metabolic problem was only limited with 7 . When compared with our own study group, a significant difference is observed in terms of PEG procedure performed in patients with metabolic diseases. This may be related with the fact that metabolic diseases are observed with a higher rate in our country because of consanguineous marriages and genetic predisposition and/or there is an active division of pediatric metabolism in our clinic. When the indications for PEG in patients with metabolic diseases were examined, it was observed that difficulty in swallowing secondary to neurologic sequela which developed as a result of primary metabolic imbalance constituted the rationale for the procedure. However, in a significant portion, the requirement of continuous presentation of energy to the body which necessitated long-term enteral feeding as in glucogen storage disease caused the patient to be referred for PEG placement. In cases where prolonged fasting can not be tolerated because of hypoglycemia as in glucogen storage disease, PEG placement and feeding by pump drop by drop while sleeping at night are helpful in preventing the common complications of the relevant diseases. In an article which investigated the morbidity and mortality of $P E G$ in children with neurologic diseases, the mean weight $Z$ score in 98 patients was found to be -3.52 before the procedure and a 1,05 increase was observed in Z score about 6 months after the procedure (6). Similar results were also found in our series. The median weight $Z$ score which was found to be -1.98 initially was observed to increase to 1.21 after a mean period of 18 months following the procedure which was a significant difference.

$P E G$ is used to prevent cachexia which may develop in relation with the primary disease or chemotherapy/ radiotherapy in hematology-oncology patients $(7,8,9,10)$. Aquino et al. (7) placed PEG in 25 children with cancer for the aim of correcting malnutrition and found PEG to be safe anf efficient in care and treatment of patients with cancer. 4 years after this study Pedersen et al. (8) reported that they placed PEG in 32 children with cancer, bone marrow transplantation was performed in 5 patients with the PEG placed, the absolute neutrophil count was below $500 / \mathrm{mm} 3$ in 7 patients (22\%) during PEG placement, but no significant complications developed in the patients in question. In a study performed in Australia in 2011, PEG was placed in 14 children with cancer and 16 infections were found in 9 
children. It was observed that infections were more frequent in children with malnutrition and no severe complication developed in any child (9). Locher et al. (11) investigated the efficiency of use of preventive PEG in adult patients with head-neck tumor. The investigators who screened 739 studies related with PEG in the literature decided that the efficiency of use of preventive PEG in adult patients with head-neck tumor could not be clearly demonstrated in the studies conducted so far. Among our patients, the number of patients with hematologic tumors or solid organ tumors is considerably limited. The reasons of the fact that we performed a limited number of PEG procedure in hematology-oncology patients may be that hematologistsoncologists did not consider malnutiriton in the patients, the patients stayed in the hospital for a short time and did not need PEG or we had inadequate physical conditions and thus the children who were immunocompromized had
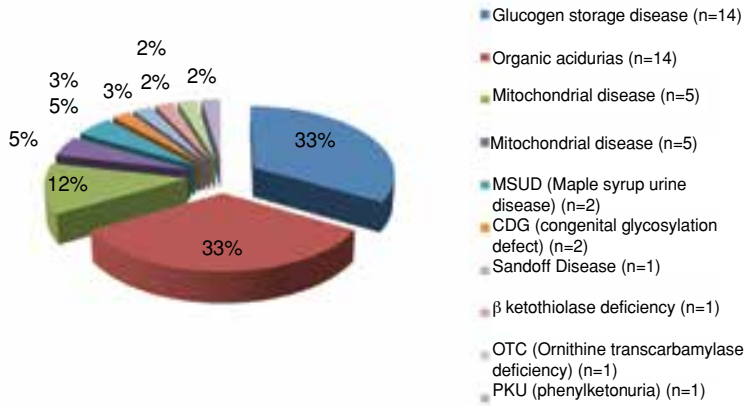

Graphic 3. Distribution of metabolic diseases predisposition to infections. However, the results reported in the above-mentioned sources suggest that PEG procedure will become a more frequently used method for the aim of decreasing the morbidity and mortality rates preventing development of malnutrition in hematology-oncology patients in our country as the consciousness about this issue increases.

When the procedures related with percutaneous endoscopic gastrostomy were examined, it was reported that the procedure of removal of PEG constituted a significant portion in the total number of procedures in large series. In the series of Srinivasan et al. (5), 49 of a total of 601 procedures were removal of PEG. In our series, only three procedures of PEG removal was performed among 104 children. The reason of this low number of PEG removal in our patients may be the fact that $P E G$ is placed frequently in chronic patients who are not expected to result in cure in our clinic. Therefore, regular PEG exchange is performed in our patients, since the ground of feeding by PEG is not eliminated.

Although the procedure of percutaneous endoscopic gastrostomy is efficient and safe, complications may be observed during or after the procedure. In a review publisehd by Fröhlich et al. (12) in 2010, the frequency of complications related with PEG procedure was reported to be in a wide range $(4.9 \%-50 \%)$. The mortality rate related with percutaneous endoscopic gastrostomy was reported to be $0.5 \%-1.2 \%$ and the morbidity rate related with percutaneous endoscopic gastrostomy was reported to range between $3 \%$ and $12 \%$. The most common complication following percutaneous endoscopic

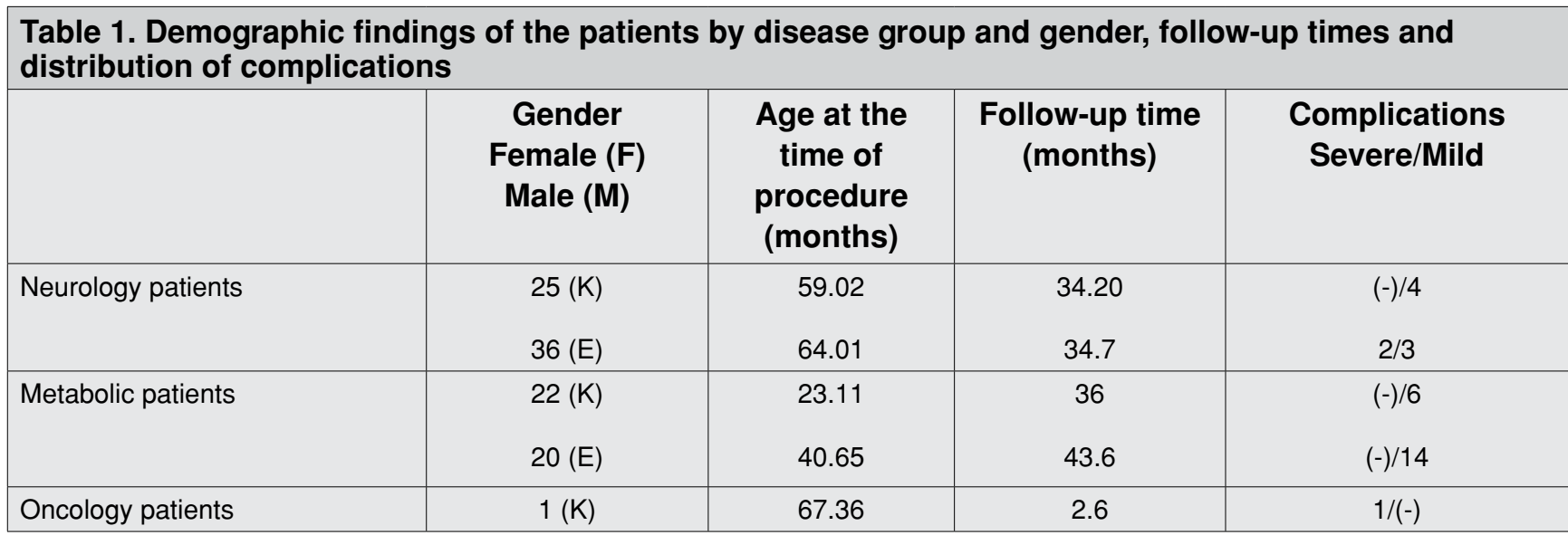

Table 2. Distribution of mild complications

\begin{tabular}{|l|c|l|c|}
\hline Standard PEG & N=6 & Button PEG & $\mathrm{N}=21$ \\
\hline Sinking of PEG disc -surgical intervention & 2 & BExplosion of Button PEG balloon - seperation of PEG tube & 13 \\
\hline Puncture, tear, cracks in PEG tube & 4 & Breaking of Button PEG tube & 6 \\
\hline & & Inability to deflate Button PEG balloon - need for endoscopy & 2 \\
\hline
\end{tabular}


gastrostomy is wound infection. It generally has a mild prognosis and regresses with intravenous antibiotic treatment. In our series, cutaneous complications related with PEG placement were observed in 13 patients and the frequency was found to be $13.2 \%$. Infection was observed in 7 of these 13 patients; Pseudomonas was grown in 6 of 10 wound swab cultures obtained form 7 patients and dual microorganisms were grown in the remaining four. The patients were cured with antibiotic treatment without problem.

When the PEG complications in our series were examined, it was observed that three significant complications developed $(2,8 \%)$ and one of these resulted in mortality $(0,96 \%)$. When compared with large series, the significant rate of complications we found in our patients was observed to be similar to the series published $(4,5,6)$. In series related with children in our country, Ulukaya Durakbaşa et al. (13) performed 26 PEG procedures in 25 children in 44 months according to the information they published in 2008 . While the mortality rate was not reported in this series, it was reported that gastrocolic fistula developed in one patient and complications necessitating re-procedure developed in three patients.

Conclusively, PEG has become an efficient feeding method which is used in different patient groups including especially metabolic diseases in our country compared to abroad and which has similar complication rates in comparison with other countries. More frequent use of PEG based on accurate grounds in a conscious way including hematology-oncology patient groups may provide convenience in treatment and care for patients, healthcare workers and institutions as well as relatives. The significant difference in weight before and after the procedure in patients who underwent PEG suggests that utilization of PEG in order to provide application of enteral feeding and efficient application of this when necessary will increase the quality of life and prolong survival in patients with malnutrition and in patients whose risk of development of malnutirition is high including especially cancer patients.

\section{Conflict of interest: None declared.}

\section{References}

1. Gauderer MW, Ponsky JL, Izant RJ Jr. Gastrostomy without laparotomy - a percutaneous endoscopic technique. J Pediatr Surg 1980; 15: 872-875.

2. Keymling M, Schlee P, Worner W. Die perkutane endoskopisch kontrollierte Gastrostomie. Dtsch Med Wochenschr 1987;112:182183.

3. Gauderer MW. Percutaneous endoscopic gastrostomy-20 years later: a historical perspective. J Pediatr Surg 2001; 36: 217-219.

4. Fortunato JE, Troy AL, Cuffari C, Davis JE, Loza MJ, OlivaHemker M, Schwarz KB. Outcome after percutaneous endoscopic gastrostomy in children and young adults. J Pediatr Gastroenterol Nutr 2010; 50: 390-393.

5. Srinivasan R, Irvine T, Dalzell M. Indications for percutaneous endoscopic gastrostomy and procedure-related outcome. J Pediatr Gastroenterol Nutr 2009; 49: 584-588.

6. Catto-Smith AG, Jimenez S. Morbidity and mortality after percutaneous endoscopic gastrostomy in children with neurological disability. J Gastroenterol Hepatol 2006; 21: 734-738.

7. Aquino VM, Smyrl CB, Hagg R, McHard KM, Prestridge L, Sandler ES. Enteral nutritional support by gastrostomy tube in children with cancer. J Pediatr 1995; 127: 58-62.

8. Pedersen AM, Kok K, Petersen G, Nielsen $\mathrm{OH}$, Michaelsen KF, Schmiegelow K. Percutaneous endoscopic gastrostomy in children with cancer. Acta Paediatr 1999; 88: 849-852.

9. Parbhoo DM, Tiedemann K, Catto-Smith AG. Clinical outcome after percutaneous endoscopic gastrostomy in children with malignancies. Pediatr Blood Cancer 2011; 56: 1146-1148.

10. Yılmaz B, Erdem D, Kemal Y. Kanser hastalarında beslenme. İç Hastalıkları Dergisi 2011; 18: 133-143.

11. Locher JL, Bonner JA, Carroll WR, Caudell JJ, Keith JN, Kilgore ML, Ritchie CS, Roth DL, Tajeu GS, Allison JJ. Prophylactic percutaneous endoscopic gastrostomy tube placement in treatment of head and neck cancer: a comprehensive review and call for evidence-based medicine. JPEN J Parenter Enteral Nutr 2011; 35: 365-374

12. Fröhlich T, Richter M, Carbon R, Barth B, Köhler H. Review article: percutaneous endoscopic gastrostomy in infants and children. Aliment Pharmacol Ther 2010; 31: 788-801.

13. Fröhlich T, Richter M, Carbon R, Barth B, Köhler H. Çocuklarda perkütan endoskopik gastrostomi (PEG) uygulamalarının endikasyonları, komplikasyonları ve sonuçlarına ilişkin bir derleme. Çocuk Cerrahisi Dergisi 2008; 22: 122-126. 